Tödliche Präsenz. Primitivismus in Hofmannsthals "Elektra"

Schneider, Sabine

DOI: https://doi.org/10.1515/9783110286670.191

Posted at the Zurich Open Repository and Archive, University of Zurich

ZORA URL: https://doi.org/10.5167/uzh-68220

Book Section

Published Version

Originally published at:

Schneider, Sabine (2012). Tödliche Präsenz. Primitivismus in Hofmannsthals "Elektra". In: Gess, Nicola. Literarischer Primitivismus. Berlin: de Gruyter, 191-210.

DOI: https://doi.org/10.1515/9783110286670.191 


\section{Tödliche Präsenz}

\section{Primitivismus in Hofmannsthals Elektra}

Es ist kein Zufall, dass die aktuellen Theoriedebatten um das neue Leitparadigma der Präsenz, wie es von Jean Luc Nancy, Hans Ulrich Gumbrecht, Martin Seel, Lambert Wiesing, Karl Heinz Bohrer, Ludwig Jäger, Sibylle Krämer und Dieter Mersch unter philosophischer, bildtheoretischer, sprachanalytischer und medientheoretischer Perspektive diskutiert wird, auf Modelle der Jahrhundertwende zurückgreifen können. ${ }^{1}$ Explizit oder implizit führen sie phänomenologische Denkmodelle ins Feld, um gegen die poststrukturalistische Verabsolutierung des linguistic turn, gegen die Prädominanz von Textualität, Zeichenregime und Sinnkultur, die aisthetische Komponente eines sinnlichen Erscheinens und die im Bedeuten nicht aufgehende körperliche Dimension materieller Leiblichkeit einzuklagen. Konnte sich die von Gumbrecht polemisch eingeführte schematische Gegenüberstellung von cartesianischen Sinnkulturen und Heideggerianischer Präsenzkultur zwar nicht durchsetzen, so findet das neue Stichwort Präsenzästhetik hingegen breiten Konsens. „Präsenz“, so resümiert Martin Seel in seiner Monographie Die Macht des Erscheinens, „erscheint in den jüngsten Theorien geradezu als die Pointe der ästhetischen Praxis und ihrer Theorie.“2 Die philosophische Ästhetik handle von nichts anderem als „,[v]on solcher Präsenzerfahrung, Präsenzgewinnung und Präsenzerzeugung [...].“3

Die theoretischen Einzelbestimmungen dieser Präsenz aber erinnern in ihrer Abgrenzung gegen die Dominanz des Symbolischen und Semiotischen in frappierender Weise an die phänomenologischen wie auch die lebensphilosophischen sprachkritischen Konzepte der frühen Moderne um 1900, die sich als Epistemo-

\footnotetext{
1 Nancy, Jean Luc: The birth to presence. Stanford 1993; Gumbrecht, Hans Ulrich: Diesseits der Hermeneutik. Die Produktion von Präsenz. Frankfurt a. M. 2004; Seel, Martin: Die Macht des Erscheinens. Texte zur Ästhetik. Frankfurt a. M. 2007; Wiesing, Lambert: Artifizielle Präsenz. Studien zur Philosophie des Bildes. Frankfurt a. M. 2005; Bohrer, Karl-Heinz: Das absolute Präsens. Die Semantik ästhetischer Zeit. Frankfurt a. M. 1994; Jäger, Ludwig: „Schauplätze der Evidenz: Evidenzverfahren und kulturelle Semantik. Eine Skizze“. In: Die Listen der Evidenz. Hg. v. Michael Cuntz u. a. Köln 2006, S. 37-52; Krämer, Sybille: „Performanz - Aisthesis. Überlegungen zu einer aisthetischen Akzentuierung im Performanzkonzept“. In: Ereignis Denken. TheatRealität, Performanz, Ereignis. Hg. v. Arno Böhler u. Susanne Granzer. Wien 2009, S. 141-168; Mersch, Dieter: Was sich zeigt. Materialität, Präsenz, Ereignis. München 2002.

2 Seel: Die Macht des Erscheinens, S. 82.

3 Ebd., S. 83.
} 
logie und Poetik der Präsenz beschreiben lassen. Präsenz in der Moderne stehe, so Martin Seel, anders als im Cartesianismus nicht für kognitive Beherrschung, sondern „,...] für Kontingenz, Augenblicklichkeit und Unverfügbarkeit - für eine Ereignishaftigkeit des Erscheinenden, das begrifflich weder gesichert werden kann noch gesichert werden soll."4 Von der Plötzlichkeit, Flüchtigkeit und der radikalen Empfindung von Zeitlichkeit als Sensibilität für die gelebte Zeit einer herausgehobenen Gegenwart ist bei Seel die Rede, von der schockartigen Unterbrechung des Zeitkontinuums, vom Grenzphänomen der Präsenz, das als unverfügbares in eine gleichzeitige Absenzstruktur gestellt ist, von der Infragestellung des Faktischen durch die Horizonteröffnung eines Möglichen, von der produktiven Verunsicherung gegenüber unseren habituellen Konzeptualisierungen, von der Bewusstwerdung der Grenzen des Sinns und schliesslich von der besonderen Intensität der Verworrenheit:

Der verworrenen, nicht an begrifflicher Distinktion und Deskription orientierten Auffassung eignet eine Intensität eigener Art. In ihr sind wir dem Hier und Jetzt der Dinge in einer Weise nahe, wie wir es in der Berufung auf wahre Repräsentationen nicht sein können. ${ }^{5}$

Der phänomenologische Philosoph Manfred Sommer hat dies für Ernst Mach und Edmund Husserl als „Evidenz im Augenblick“6 beschrieben, als anticartesianische paradoxe Logik einer Evidenz im Verworrenen, auf der sprachabgewandten dunklen Rückseite der symbolischen Ordnung, an der Schwelle des Bewusstseins und der intentionalen Aufmerksamkeit. Es ist dies jene Schwelle, die der Mediziner Ernst Kretschmer 1922 die „Sphäre“ des Bewusstseins nennen sollte, an deren dezentriertem Standort er den Ort kreativer Prozesse wie auch pathologischer Konstellationen situierte. ${ }^{7}$

Und auch Dieter Mersch begibt sich in seiner Fundamentalkritik am universalen Textbegriff der Dekonstruktion auf die Suche nach den Grenzen des Symbolischen. Auch er beruft sich wie die anderen Vertreter einer Präsenzästhetik auf die Irreduzibilität des phänomenologischen Erscheinens, auf die deiktische Komponente eines sich Zeigens, das im Sagen nicht aufgeht. Was sich zeigt, so Mersch, ist das „Quod“ eines „dass“, das primordial vor dem „Quid“ „als etwas“ erscheint, ist das Erscheinen eines Seins vor dem Bedeuten, der Einbruch eines

\footnotetext{
4 Ebd., S. 82.

5 Ebd., S. 89. Vgl. das Kapitel „Über den kulturellen Sinn ästhetischer Gegenwart - mit Seitenblicken auf Descartes“, S. 82-94.

6 Sommer, Manfred: Evidenz im Augenblick. Eine Phänomenologie der reinen Empfindung. Frankfurt a. M. 1996.

7 Kretschmer, Ernst: Medizinische Psychologie. Ein Leitfaden für Studium und Praxis. Leipzig 1922, S. $66 \mathrm{f}$.
} 
Ereignisses, der als „Entzug oder Riß im Symbolischen“ wahrgenommen wird und als „Widerfahrnis“ nicht intentional gesucht werden kann. ${ }^{8}$ Präsenz ist somit bei Mersch wie bei Seel eine Grenzerfahrung. Die „Fülle des Ereignens“ setze die „Leere des Sinns“ voraus, sie erfordere „Grenzgänge“, „Limitationen“, wie sie für die Happenings der Gegenwartskunst symptomatisch seien. ${ }^{9}$ Für diese liminalen Erfahrungen am Rand des Symbolischen setzt Mersch den Begriff des Ekstatischen und damit verbunden den der Aura ein: „Die Aura duldet keinen Verweis. Sie ist überhaupt nicht lesbar: Sie widerfährt. Bevorzugt widerfährt sie dort, wo sich die Aufmerksamkeit offenhält für ein Ekstatisches. “10

All diese Bestimmungen zum Paradigma der Präsenz sind ausnahmslos bereits in Hofmannsthals Poetik formuliert, wie sie mit dem spektakulären Auftakt des Chandosbriefs 1902 und seiner Absage an den „Prunk der Worte“, „die geläufigen Bilder“ und das „Gehege der rhetorischen Kunststücke“ seine poetologischen Reflexionen und die literarische Produktion bis zum Ersten Weltkrieg bestimmen sollte. ${ }^{11}$ Die guten Augenblicke des an der Rationalität der Sprache irre gewordenen Lord Chandos sind plötzliche Illuminationen angesichts eines Einbruchs von Seinsfülle „stummer Wesenheit“, deren Erlebnis als emphatische Gegenwart beschrieben wird. ${ }^{12}$ „Alles war in mir [...]“", beschreibt Chandos die aufflammende Vision der sterbenden Ratten, „[...] und es war Gegenwart, die vollste erhabenste Gegenwart." ${ }^{13}$ Es sind keine Repräsentationen, weder wird zwischen Vorstellung und Realität unterschieden, noch unterscheidet sich das Bewusstsein von den Bewusstseinsinhalten. Gerade die Differenzlosigkeit des Ineinanderfliessens macht die übernatürliche fraglose Evidenz dieser Illuminationen aus. Es sind ekstatische Grenzerfahrungen von „Dumpfheit“, „,verworrenste Gedanken“ 14 bei abgesenktem Bewusstseinsstand, Momente, in denen das Fluidum von Wachen und Schlafen in eins geflossen ist, dem rationalen Bereich des diskursiven Denkens und dem kulturellen Bedeutungskosmos von Sprache und Schrift entzogen. Die Sprache als Repräsentation und als differenzierendes Zeichensystem ist jenen stummen Momenten der Differenzlosigkeit gegenüber

8 Mersch: Was sich zeigt, S. $373 \mathrm{ff} .$, Zitate S. 375.

9 Ebd., S. 375.

10 Ebd.; vgl. auch Mersch, Dieter: Ereignis und Aura. Untersuchungen zu einer Ästhetik des Performativen. Frankfurt a. M. 2002.

11 Hofmannsthal, Hugo von: „Ein Brief“. In: ders.: Sämtliche Werke. Kritische Ausgabe. Veranstaltet vom Freien Deutschen Hochstift. Hg. v. Rudolf Hirsch u.a. Bd. XXXI. Erfundene Gespräche und Briefe. Hg. v. Ellen Ritter. Frankfurt a. M. 1991, S. 45 f. Im Folgenden Sigle SW XXXI.

12 Ebd., S. 53.

13 Ebd., S. 51.

14 Ebd., S. 52. 
hoffnungslos nachträglich und defizient, so die Anordnung des kleinen Sprachdramas, das zum kanonischen Text der sprachkritischen Moderne werden sollte. Was Hofmannsthal in Ad me ipsum rückblickend als „Situation des Mystikers ohne Mystik “15 bezeichnen sollte, jene Poetik der visionären und ekstatischen Entgrenzung durch halluzinative Techniken der Präsentierung, ist ein aus der Skepsis gegenüber der abstrakten Repräsentationsfunktion der Sprache motiviertes Experiment mit dem, was Hofmannsthal „Vivizierung“ der symbolischen Systeme nennt. ${ }^{16}$ Intendiert ist eine Poetik der Präsenz anstelle der Repräsentation, eine Vertauschungs- und Entgrenzungslogik zwischen Abbild und Urbild, der Einzug von Differenzen, das Oszillieren der Zeichen zwischen Repräsentation und imaginativer Anwesenheit, die Erfahrung von Partizipation und ekstatischer Teilhabe anstelle blosser Symbolisierung.

Diese Zeichenutopie unter dem Fanal der Präsenz ist die Systemstelle für den Primitivismus in Hofmannsthals Poetik. Am Gespräch über Gedichte, im selben Jahr wie das Drama Elektra entstanden, lässt sich dieser Zusammenhang vielleicht am explizitesten zeigen. ${ }^{17}$ Der Stellvertreterlogik des sprachlichen Zeichens als rein rhetorische Übertragung der Metapher setzt das Gespräch ein Unmittelbarkeitsgebot entgegen, die Sache selbst zu setzen: „Niemals setzt die Poesie eine Sache für eine andere, denn es ist gerade die Poesie, welche fieberhaft bestrebt ist, die Sache selbst zu setzen [...]." ${ }^{\text {"18 }}$ Nicht Stellvertretung und Abwesenheit als Signa blossen Bedeutens, sondern Anwesenheit und Sein setzt die mit den Attributen des Magischen versehene lyrische Poesie: „[W]as niemals da war, nie sich gab, jetzt ist es da, jetzt gibt es sich, ist Gegenwart, mehr als Gegenwart [...]." ${ }^{\text {19 }}$ Die Zauberkraft der Poesie, Gegenwart herbeizuzwingen, macht den Dichter zum Schamanen. Im Vortrag Der Dichter und diese Zeit von 1908 nennt Hofmannsthal

15 Hofmannsthal, Hugo von: „Ad me ipsum“. In: ders.: Gesammelte Werke in zehn Einzelbänden. Reden und Aufsätze III (1925-1929). Hg. v. Bernd Schoeller in Beratung mit Rudolf Hirsch. Frankfurt a. M. 1979, S. 601. Im Folgenden Sigle RuA III.

16 Hofmannsthal, Hugo von: „Der Dichter und diese Zeit“. In: ders.: Sämtliche Werke.

Kritische Ausgabe. Veranstaltet vom Freien Deutschen Hochstift. Hg. v. Rudolf Hirsch u. a. Bd. XXXIII. Reden und Aufsätze 2. Hg. v. Konrad Heumann und Konrad Ritter. Frankfurt a. M. 2011, S. 130. Im Folgenden Sigle SW XXXIII. Hofmannsthal übernimmt das Novalis-Zitat von Walter Pater: The Renaissance. London, New York 1900, S. 236: „Philosophiren, says Novalis, ist dephlegmatisiren, vivificiren“.

17 Vgl. Schneider, Sabine: „Poetik der Illumination. Hugo von Hofmannsthals Bildreflexionen im Gespräch über Gedichte“. In: Zeitschrift für Kunstgeschichte 71 (2008), Heft 3, S. 389404.

18 Hofmannsthal: „Das Gespräch über Gedichte“. In: SW XXXI, S. 77.

19 Ebd., S. 86. 
den Dichter einen „Schattenbeschwörer ohne Maß“. ${ }^{20}$ An anderer Stelle spricht er vom geisterhaften Licht und dem feurigen Rauch, aus dem der Dichter die Erscheinungen erstehen lasse. ${ }^{21}$ Der Dichter, von dem es heisst: „In ihm oder nirgends ist Gegenwart“, übt einen Geisterzwang aus. ${ }^{22}$ Das Bild der sich am geopferten Blut sättigenden und dadurch zu neuem Leben gebrachten Schattenseele taucht immer wieder in Hofmannsthals poetologischen Skizzen auf. Es steht auch am Anfang seiner Auseinandersetzung mit dem Elektra-Stoff. Man müsse, so hält es eine Notiz von 1903 unter der Überschrift Vertheidigung der Elektra fest, „[a]us dem Blut wieder Schatten aufsteigen lassen. “23

Die Vorstellung des Eidolon, der Schattenseele, übernahm Hofmannsthal aus Erwin Rohdes Standardwerk Psyche. Seelencult und Unsterblichkeitsglaube der Griechen, einer ethnologisch-primitivistischen Perspektive auf die griechische Antike in vorhomerischer Zeit. ${ }^{24}$ Bereits 1894 finden sich Notizen, die von der Sprachbeherrschung des Dichters sprechen als „Götzendienst, Anbetung eines eidolon, Sinnbildes, das einmal für einen Menschen lebendig war, Mirakel gewirkt hat, durchflammende Offenbarung des göttlichen Geheimnisses der Welt.“25 Denkt eine Notiz zum Gespräch über Gedichte über das „poetische Organ“ in uns nach, dessen Funktion es ist, „[...] daß es vor uns ewige Gegenwart herzaubert“, ${ }^{26}$ so spricht in der dialogischen Anordnung des fiktiven Gesprächs die avanciertere Figur Gabriel im selben Sinn von der „Bezauberung“ durch das poetische Symbol, welches „uns so furchtbar nahe“ auf den Leib rücke wie in archaischer Zeit, als „[i] m dunstigen Dunkel, unter Schreien, unter taumelndem Fackelschein [...] auf einmal Aphrodite aus dem Purpurschaum geboren [...]“" wurde. ${ }^{27}$

20 Hofmannsthal: „Der Dichter und diese Zeit“. In: SW XXXIII, S. 140. Zu Hofmannsthals Dichterkonzept in diesem Vortrag vgl. Schneider, Sabine: „Die Welt der Bezüge.

Hofmannsthal zur Autorität des Dichters in seiner Zeit“. In: Colloquium Helveticum 41 (2010),

S. $203-221$.

21 Hofmannsthal: „Einleitung zur neuen Balzac-Ausgabe“. In: SW XXXIII, S. 176; ders.: „Dichter und Leben“. In: ders.: Gesammelte Werke in zehn Einzelbänden. Reden und Aufsätze I (1891-1913). Hg. v. Bernd Schoeller in Beratung mit Rudolf Hirsch. Frankfurt a. M. 1979,

S. 235. Im Folgenden Sigle RuA I.

22 Hofmannsthal: „Der Dichter und diese Zeit“. In: SW XXXIII, S. 138.

23 Hofmannsthal: „Vertheidigung der Elektra“. In: SW XXXIII, S. 222.

24 Rohde, Erwin: Psyche. Seelencult und Unsterblichkeitsglaube der Griechen. 2 Bde. 3. Aufl. Tübingen, Leipzig 1903, Kap. ,Seelenglaube und Seelencult in den homerischen Gedichten“; Kap. ,Der Seelencult. I. Cultus der chthonischen Götter; II. Pflege und Verehrung der Todten“. 25 Hofmannsthal: „Aufzeichnungen aus dem Nachlass 1894-95“. In: RuA III, S. 390.

26 Hofmannsthal: „Das Gespräch über Gedichte. Varianten und Erläuterungen.“ In: SW XXXI, S. 324.

27 Ebd, S. 81 u. S. 83. 
Was Hofmannsthal mit diesem Beispiel der Entstehung der Aphrodite aus einer ekstatisch-dionysischen Kelterszene anzitiert, ist die ethnologische Metapherntheorie der Jahrhundertwende. Sie sieht die analogische Denkform der Metapher als primitive Logik an und situiert sie in einem primitiven mythischen Lebensvollzug. In seiner Rezension von Alfred Bieses Philosophie des Metaphorischen von $1894,{ }^{28}$ in der alle wichtigen Theoreme der Diskussion zusammengefasst sind, spricht Hofmannsthal vom „metaphernbildenden Trieb in uns“, von „der unheimlichen Herrschaft, die die von uns erzeugten Metaphern rückwirkend auf unser Denken ausüben“, und von „der unsäglichen Lust, die wir durch metaphorische Beseelung aus toten Dingen saugen.“ Was die Metapher an Wirklichkeit schafft, ist „ein mystische[r] Vorgang“, „leuchtend und real““. ${ }^{29}$ Eine Notiz zum Gespräch über Gedichte macht deutlich, dass jene „orphische [...] Sinnlichkeit“, welche uns Modernen weitgehend abhanden gekommen sei, auf die Animismustheorie des englischen Kulturanthropologen Edward B. Tylor rekurriert: „Uns gehen nicht mehr feuchte Wolken in Gestalten über Vogelanflug ist uns nicht Anhauch von Geistern“. ${ }^{30}$ Tylor sah in der analogischen Denkform eine primitive Anschauungsform, die einem unbewusst wirkenden, atavistischen Kausaltrieb entstamme und das primitive Bewusstsein zwinge, einer unbekannten Erscheinung eine scheinbar verwandte anthropomorphe Ursache unterzulegen. ${ }^{31}$ Dieses Grundaxiom des Primitivismus geht diskursgeschichtlich eine Konjunktion mit der Sinnesphysiologie ein, denn diese Analogien wurden als endogene Bilder, als Halluzinationen aufgefasst. Der mythische Kausaltrieb Tylors ist eine Übertragung der von Hermann Helmholtz vertretenen sinnesphysiologischen Lehre der in-

28 Biese, Alfred: Die Philosophie des Metaphorischen. In Grundlinien dargestellt. Hamburg, Leipzig 1893.

29 Hofmannsthal: „Philosophie des Metaphorischen“. In: RuA I, S. 192. Vgl. Riedel, Wolfgang: „Arara ist Bororo oder die metaphorische Synthesis“. In: Anthropologie der Literatur. Poetogene Strukturen und ästhetisch-soziale Handlungsfelder. Hg. v. Rüdiger Zymner u. Manfred Engel. Paderborn 2004, S. 220-240; ders.: „Archäologie des Geistes. Theorien des wilden Denkens um 1900“. In: Das schwierige neunzehnte Jahrhundert. Hg. v. Jürgen Barkhoff u.a. Tübingen 2000, S. 467-485; Schneider, Sabine: „Das Leuchten der Bilder in der Sprache. Hofmannsthals medienbewusste Poetik der Evidenz".In: HofmannsthalJahrbuch 11 (2003), S. 209-248.

30 Hofmannsthal: „Das Gespräch über Gedichte. Varianten und Erläuterungen“. In: SW XXXI, S. 327.

31 Tylor, Edward B.: Primitive Culture. Reasearch into the Development of Mythology, Philosophy, Religion, Art, and Custom. 2 Bde. London 1871; dt.: Die Anfänge der Cultur. Untersuchungen über die Entwicklung der Mythologie, Philosophie, Religion, Kunst und Sitte. Unter Mitwirkung des Verfassers ins Deutsche übertragen v. J.W. Spengel u. Fr. Poske. 2 Bde. Leipzig 1873. 
duktiven „unbewussten Schlüsse“ in die Kulturanthropologie. ${ }^{32}$ Diese Halluzinationen erregten, so Tylor mit Helmholtz, die Sehsinnsubstanz. Kritiklos für völlig evident erwiesen seien sie für das primitive Bewusstsein Wirklichkeiten, nicht Repräsentationen: „Analogien, welche in unsern Augen nichts als Phantasien sind, waren für Menschen vergangener Zeiten Wirklichkeit. Sie konnten die Flamme ihre noch unverzehrte Beute mit Feuerzungen belecken sehn. “33

Die Verbindung von sinnesphysiologischen, ethnologischen und sprachphilosophischen Theoremen in der ethnologischen Theorie vom mythischen Bewusstsein als Ursprung der Metapher hatte Friedrich Nietzsche in einer für die Jahrhundertwende folgenreichen Weise in seinen Aphorismen zur Logik des Traums in Menschliches, Allzumenschliches ausformuliert. Das analogische Denken, wie es sich im Bilderdenken des Traums und in der Metapher manifestiert, ist für Nietzsche, mit Tylor gedacht, ein primitives survival in der Moderne. In Zuständen „beeinträchtigt[er] [...] Gehirnfunction“ gewinnt die wilde Semiose dieser primitiven Denkform ihre alte Macht zurück. „Willkürlich und verworren [...]“ verwechsle das analogische Denken „[...] fortwährend die Dinge auf Grund der flüchtigsten Ähnlichkeiten: aber mit derselben Willkür und Verworrenheit dichteten die Völker ihre Mythologien [...].“34 In jenen Traum- und Halbtraumvorstellungen von halluzinatorischer Deutlichkeit kehrten, so Nietzsche, „Zustände früherer Menschheit“ zurück, „in der die Hallucination ausserordentlich häufig war und mitunter ganze Gemeinden, ganze Völker gleichzeitig ergriff. “35 An dieses ältere Menschentum, ein „Urphänomen“, demgegenüber das „schärfere logische Denken, das Strengnehmen von Ursache und Wirkung“ eine spätere Entwicklung ist, erinnere nach Nietzsche auch der Dichter, der Künstler mit seinem Analogienzauber. ${ }^{36}$

Hofmannsthal greift wie Nietzsche auf die ethnologisch argumentierende Metapherntheorie zurück, um der dichterischen Sprache die Evidenz mythischer

32 Helmholtz, Hermann: Handbuch der physiologischen Optik. Leipzig 1867, S. 194 u.

S. 443.

33 Tylor: Die Anfänge der Cultur, Bd. 1, S. $293 \mathrm{f}$.

34 Nietzsche, Friedrich: „Aph. 12. Traum und Cultur“. In: ders.: Sämtliche Werke. Kritische Studienausgabe in 15 Bänden. Hg. v. Giorgio Colli und Mazzino Montinari, Bd. 2.

Menschliches, Allzumenschliches I und II. Neuausgabe. München 1999, S. 31 f. Im Folgenden Sigle KSA 2. Sowie ders.: ebd., Bd. 7. Nachgelassene Fragmente 1869-1874, S. $483 \mathrm{f}$.

35 Nietzsche, Friedrich: „Aph. 12. Traum und Cultur“. In: KSA 2, S. $31 \mathrm{f}$. Vgl. Treiber, Hubert: „Zur Logik des Traumes bei Nietzsche. Anmerkungen zu den Traum-Aphorismen aus Menschliches, Allzumenschliches“. In: Nietzsche-Studien 23 (1994), S. 1-41; Pfotenhauer, Helmut u. Sabine Schneider: Nicht völlig Wachen und nicht ganz ein Traum. Die Halbschlafbilder in der Literatur. Würzburg 2006.

36 Nietzsche, Friedrich: „Aph. 13. Logik des Traumes“. In: KSA 2, S. 34 f. 
Welterfahrung zuschreiben zu können. Dass die Metapher blitzartig, mit elementarer Gewalt über uns komme, dass sie mit einem zerebralen Erregungsgeschehen verbunden sei und als solch ekstatischer Einbruch unverfügbar, dem selbstgewissen Sprachspiel entzogen sei, wie Hofmannsthal in dem kleinen Text über Die Philosophie des Metaphorischen schreibt, gehört in diesen Kontext. ${ }^{37}$ Und ein zweiter primitivistischer Erklärungszusammenhang wird im Gespräch über Gedichte bemüht, um dem Sprachzeichen, dem in der Moderne durch symbolistische Raffinessen schal gewordenen dichterischen Wort, die „Wucht und Fremdheit“"38 archaischer Bildmacht zurück zu erstatten. Hofmannsthal leitet das dichterische Symbol aus dem Kultus des Opfers her, eine Herleitung, die ihm Adorno als gewaltverherrlichenden Barbarismus übel genommen hat. ${ }^{39}$ Was Hofmannsthal jedoch hier erprobt, ist nicht inhaltlich, sondern semiologisch motiviert. Die Faszination gilt einem partizipatorischen, statt repräsentativen Zeichenmodell, gilt der Kraft der Vermischung und Verwechslung in einer kontagiösen Situation, also der Übertragungsmagie im Sinne James George Frazers: ${ }^{40}$

[...] auf einmal zuckte dem Tier das Messer in die Kehle, und das warme Blut rieselte zugleich an dem Vließ des Tieres und an der Brust, an den Armen des Menschen hinab: und einen Augenblick lang muß er geglaubt haben, es sei sein eigenes Blut [...]: er muß, einen Augenblick lang, in dem Tier gestorben sein, nur so konnte das Tier für ihn sterben. Daß das Tier für ihn sterben konnte, wurde ein großes Mysterium, eine große geheimnisvolle Wahrheit. Das Tier starb hinfort den symbolischen Opfertod. ${ }^{41}$

Dass Hofmannsthal hier von Symbol und nicht von Metapher spricht, verweist auf einen weiteren Intertext dieser Diskussion. Friedrich Theodor Vischer hatte in dem Aufsatz Das Symbol eine mythische Denkform von einer ästhetischen unterschieden. In der mythischen, so Vischer, wurden Bild und Bedeutung unbewusst identifiziert. Wie nach ihm Hofmannsthal exemplifiziert er die mythische Alter-

37 Hofmannsthal: „Philosophie des Metaphorischen“. In: RuA I, S. $192 \mathrm{f}$.

38 Hofmannsthal: „Die Briefe des Zurückgekehrten“. In: SW XXXI, S. 174.

39 Adorno, Theodor W.: „George und Hofmannsthal. Zum Briefwechsel: 1891-1906“. In: ders.: Prismen. Kulturkritik und Gesellschaft. Berlin, Frankfurt a. M. 1955, S. 232-282, hier S. $277 \mathrm{f}$.: „Diese blutrünstige Theorie des Symbols, welche die finsteren politischen Möglichkeiten der Neuromantik einbegreift, spricht etwas von ihren eigentlichen Motiven aus [...]. Im Namen der Schönheit weiht er sich der übermächtigen Dingwelt als Opfer.“ Vgl. Wellbery, David E.: „Die Opfer-Vorstellung als Quelle der Faszination. Anmerkungen zum Chandos-Brief und zur frühen Poetik Hofmannsthals“. In: Hugo von Hofmannsthal. Neue Wege der Forschung. Hg. v. Elsbeth Dangel-Pelloquin. Darmstadt 2007, S. 186-212. 40 Vgl. Frazer, James Georges: Der goldene Zweig. Das Geheimnis von Glauben und Sitten der Völker (engl. 1894). Aus dem Englischen von Helen von Bauer. Reinbek 1989.

41 Hofmannsthal: „Das Gespräch über Gedichte“. In: SW XXXI, S. 80 f. 
native zur blossen Stellvertreterlogik des Zeichens am Opfer. Hier sei in einer partizipatorischen Auffassung die Grenze des Zeichens im Ineinanderdenken von Bild und Bedeutung aufgehoben. ${ }^{42}$

Dass Hofmannsthal im Gespräch über Gedichte für seinen elementar leiblichen Begriff von Magie auf den Opferkult rekurriert, zeigt die Bedeutung der Performanz für dieses dichterische Präsenzkonzept. Insofern ist es folgerichtig, dass er mit dem Drama Elektra, zeitgleich entstanden mit dem Gespräch über Gedichte, die Bühne als Ort für die performative, auf halluzinative Weise wirklichkeitserzeugende Macht der Bilder wählt. In der Elektra herrscht eine geisterhafte Präsenz der Bilder, von der traumatischen Fixierung der Hysterikerin und einem archaischen Geisterbann aus Schatten und Lichtflecken beschworen. ${ }^{43}$ Unterstützt wird sie von einer mythisierenden, die animistischen Projektionen des Auges affirmierenden Lichtregie. In der Elektra greift der archaische Bilderdienst auf die Struktur des ganzen Dramas über, mit tödlicher Konsequenz. Was in diesem Drama bis zur letzten Konsequenz getrieben und in seiner radikalen Konsequenz und seinen Risiken vorgeführt wird, ist die Poetik der Präsenz unter den Vorzeichen visionärer Grenzüberschreitung. Ich lese also die Elektra auch als poetologischen Text, in dem Hofmannsthal die Konsequenzen seiner eigenen Präsenzpoetik kritisch hinterfragt. In der Elektra wird jene „Welt der Bezüge“ als Logik analogischer Verwechslungen und Vermischungen installiert, in der Ähnliches das Ähnliche in wilder Semiose aufruft, die Hofmannsthal im Gespräch über Gedichte und im Vortrag Der Dichter und diese Zeit als poetisches Prinzip proklamiert. ${ }^{44}$ Wenn der Dichter als „Schattenbeschwörer ohne Maß“45 dem Bann seiner visionären Bilder ausgeliefert wird, die ihn zum entrückten Schamanen einer alle Distanz absorbierenden Gegenwart machen, dann ist Elektras geisterbeschwörender Bilderbann, ihr Wachträumen im Hypnoid von poetologischer Relevanz. Es steht also in der Elektra das poetologische Konzept der Präsenz zur Disposition. Das ist die Experimentalanordnung, unter der Hofmannsthals Rückgriff auf den antiken Mythos steht und das sind auch die Vorzeichen für die primitivistische Bearbeitung der sophokleischen Tragödie durch Hofmannsthal. ${ }^{46}$

42 Vischer, Friedrich Theodor: „Das Symbol“. In: ders.: Altes und Neues. Stuttgart 1889, S. $290-342$, hier S. $279 \mathrm{f}$.

43 Zur hysterischen Gleichzeitigkeit als Zeitstruktur des Dramas vgl. Vogel, Juliane: „Priesterin künstlicher Kulte. Ekstase und Lektüren in Hofmannsthals Elektra“. In: Tragödie Idee und Transformation. Hg. v. Hellmut Falshar. Stuttgart u. a. 1997, S. 287-306.

44 Hofmannsthal: „Der Dichter und diese Zeit“. In: SW XXXIII, S. 138.

45 Ebd.: S. 140.

46 Die Präsenzthese hat für die Elektra erstmals Bohrer vertreten: Bohrer, Karl-Heinz: „Die Wiederholung des Mythos als Ästhetik des Schreckens. Hugo von Hofmannsthals 
Die kritische Ausgabe reproduziert einige wenige Aufzeichnungen zum Entstehungsprozess der Elektra. Aus ihnen wird ersichtlich, dass es Hofmannsthal genau um jenen primitivistischen Akzent bei der Bearbeitung des Stoffes geht. „Als Stil schwebte mir vor, etwas gegensätzliches zur Iphigenie zu machen [...]“, schreibt er im Rückblick 1904. ${ }^{47}$ Doch nicht nur gegen das klassizistische Griechenlandbild der Goethezeit wendet sich seine Adaption des Mythos, sondern auch gegen die Aktualisierung unter den triebtheoretischen Vorzeichen der Psychoanalyse, wie sie Hermann Bahr in seinem Dialog vom Tragischen 1904 proklamierte. ${ }^{48}$ Während letzterer in der Rationalität von Hellas nach den hysterischen Symptomen einer verdrängten Triebstruktur fahndete, gilt Hofmannsthals Interesse nicht einer Bestätigung der psychoanalytischen Theoreme. In diametraler Umkehrung des in der Psychoanalyse angelegten Ikonoklasmus, also dem Absprechen der traumatischen Reminiszenzen durch das klärende Wort, geht es Hofmannsthal gerade um die Inszenierung jener machtvollen Gegenwart der visionären Phänomene und ihre Eigenlogik. ${ }^{49}$ Dieses halluzinierende Bilderwesen wird als Atavismus der Moderne aufgefasst, in dem die modernsten neurologischen Naturwissenschaften, die Hysteriestudien Breuers und Freuds, mit Nietzsche gesprochen, auf „dieses uralte Stück Menschenthum in uns“ stoßen, das als survival aus primitiven Zeiten mit befremdlicher Wucht in das Zeitalter der modernen Neurosen hereinragt. ${ }^{50}$ Die machtvolle Gegenwart der wiederkehrenden Bilder ist der Schnittpunkt, auf den hin Hofmannsthal auch seine beiden Quellenkomplexe, die psychopathologischen und die ethnologischen, befragt. In diesem Sinn ist der unter der Überschrift Vertheidigung der Elektra 1903 notierte

Nachdichtung von Sophokles’ Elektra“. In: ders.: Das absolute Präsens. Die Semantik ästhetischer Zeit. Frankfurt a. M. 1994, S. 63-92. Vgl. ferner meinen Vorschlag einer semiologischen Lektüre: Schneider, Sabine: „Helldunkel - Elektras Schattenbilder oder die Grenzen der semiotischen Utopie“. In: dies.: Verheißung der Bilder. Das andere Medium in der Literatur um 1900. Tübingen 2006, S. 342-368.

47 Hofmannsthal, Hugo von: „Elektra. Zeugnisse“. In: ders.: Sämtliche Werke. Kritische Ausgabe. Veranstaltet vom Freien Deutschen Hochstift. Hg. v. Rudolf Hirsch u.a. Bd. VII Dramen 5. Hg. v. Klaus E. Bohnenkamp und Mathias Mayer. Frankfurt a. M. 1997, S. 400. Im Folgenden Sigle SW VII.

48 Bahr, Hermann: Dialog vom Tragischen. Berlin 1904.

49 Die „talking cure“ der Psychoanalyse beruht auf dem therapeutischen Prinzip, dass sie der Energie der „vor dem inneren Auge“ auftauchenden Bilder „den Ablauf durch die Rede gestattet“ und sie damit „abträgt“. Breuer, Josef u. Sigmund Freud: Studien über Hysterie (1895). Hg. v. Stavros Mentzos. Frankfurt a. M. 2000, S. 297, S. 40 u. S. 271. Zum Ikonoklasmus der Psychoanalyse vgl. Schneider, Florian: „Augenangst? Die Psychoanalyse als ikonoklastische Poetologie“. In: Hofmannsthal-Jahrbuch 11 (2001), S. 197-240.

50 Nietzsche: „Aph. 13. Logik des Traums“. In: KSA 2, S. 33. 
Satz zu verstehen: „Wir müssen uns den Schauer des Mythos neu schaffen. Aus dem Blut wieder Schatten aufsteigen lassen. “51

Die Anstreichungen in Hofmannsthals Handexemplaren der beiden Hauptquellen, Freuds und Breuers Studien über Hysterie einerseits und Erwin Rohdes Psyche. Die Seelenvorstellungen der Griechen andererseits, zeigen dieses manifeste Interesse an bildmagischen Phänomenen. ${ }^{52}$ Hofmannsthals Anmerkungen im Handexemplar gelten dem ersten Kapitel des ersten Teils, in dem Rohde auf die archaischen survivals in Homers Welt anhand der primitiven Seelenvorstellung eingeht. Was er als von der Rationalität der olympischen Epoche überwundene Vorstellungen wieder ins Blickfeld rückt, ist eine Welt des magischen Geisterzwangs, der geisterhaften Präsenz der Toten und der chthonischen Opferbräuche zu ihrer Bannung oder Beschwörung. Es ist nicht „die homerische helle Welt“, die „befreit [ist] von Nachtgespenstern“ und in der „auch die Nacht [...] die entflogenen Seelen der Verstorbenen nicht frei“ gibt. ${ }^{53}$ Deren räumliche Gegenwart und ihr Einwirken auf das Reich der Lebenden macht diese zu einem Zwischenreich zwischen Leben und Tod, in dem vielfach Unentscheidbarkeit zwischen beiden Sphären herrscht. Eine Zeichenvorstellung kommt hier zum Tragen, die nicht säuberlich geschieden ist in die binäre Logik reiner Stellvertretung. Stattdessen herrschen im chthonischen Ahnenkult Kontaminationen, kontagiöse Vermischungen und Machtübertragungen zwischen Abbild und Urbild. Auch in Hofmannsthals Drama gilt eine solche Ununterscheidbarkeit zwischen der Sphäre der Lebenden und der Toten, in der die Oppositionen kollabieren und in der die Doppeldeutigkeit von Gesichtern und Geschichten verunklärt wird. ${ }^{54}$

Mit Elektras großer Opfervision am Eingang, die eine performative Umsetzung von Rohdes Schilderung der blutrünstigen Begräbnisriten des Achill für Patroklos aus dem 23. Gesang der Ilias ist, setzt das Drama mit einer bewussten Archaisierung der „klassischen“ sophokleischen Vorlage ein, unter Rückgriff auf die von Rohde als atavistische survivals identifizierten Seelenkulte, in denen nach Rohde

51 Hofmannsthal: „Vertheidigung der Elektra“. In: SW XXXIII, S. 222.

52 Breuer, Josef u. Sigmund Freud: Studien über Hysterie. Leipzig, Wien 1895; Rohde: Psyche. Beide Werke finden sich in Hofmannsthals Bibliothek im Freien Deutschen Hochstift. Die Anstreichungen zu Breuer/Freud beziehen sich auf die Krankengeschichten der Anna 0. und Emmy v. N. und unterstreichen den Begriff „Privattheater“ sowie auf den Teil „Theoretisches“ von Breuer, der die halluzinogene Struktur erläutert. Die Anstreichungen zu Rohde beziehen sich auf das erste Kapitel im ersten Band zum Seelenkult mit der Vorstellung der Eidola sowie auf das zweite Kapitel im zweiten Band zum thrakischen Dionysoskult mit seinen Ekstasetechniken.

53 Rohde: Psyche, Bd. 1, S. 11.

54 Hofmannsthal: „Elektra“. In: SW VII, S. 86. 
„uralte, längst gebändigte Rohheit ein letztes Mal hervorbricht. “55 Im zweiten Teil von Rohdes Standardwerk, geht dieser, ebenfalls aufmerksam annotiert von Hofmannsthal, auf die schamanischen Trancetechniken einer orphischen Erregungskultur ein, in der Ekstasetechniken wie „Ekstatische Mantik, Kathartik und Geisterzwang“ der „Ueberreizung der Empfindung bis zu visionären Zuständen“ dienen. ${ }^{56}$ Kulturelle Techniken wie der Dreh-Tanz der Derwische oder der Fackeltanz der Mänaden auf den thrakischen Bergen (beide Tanzformen werden in Hofmannsthals Elektra eingesetzt) dienen nach Rohde der Ekstase, um „in Berührung treten zu können“ mit „Wesen einer höheren Ordnung, mit dem Gotte und seinen Geisterschaaren“. ${ }^{57}$ Es geht um Berührung und Teilhabe, um Präsenz und nicht um symbolische Stellvertretung. Rohde zieht selbst schon psychopathologische Erklärungen für diese dionysischen Feste mit heran, die Verbindung von Kulturanthropologie und Hysterie lag für Hofmannsthal sozusagen auf der Hand. Auch Freud und Breuer bezeichneten die therapeutische Methode der „talking cure“ als „Katharsis“ und riefen für die psychohygienische Säuberung des inneren „Gesichtsfeld[s]“, die Erledigung der „Schreckbilder“ durch die Distanzierungsleistung der Sprache den Kontext archaischer Seelenvorstellungen auf. ${ }^{58}$ Sobald der Patient die Bewusstwerdung durch Versprachlichung geleistet hat, schreibt Freud in dem Kapitel Zur Psychotherapie der Hysterie, „[...] schwindet das Bild, wie ein erlöster Geist zur Ruhe eingeht. “59 Das ausgesprochene Wort ist somit der Seelengeleiter, der die eidola der im hypnoiden Wachtraum der Anna O. zurückgekehrten Bilder des toten Vaters kraft eines „magisch zwingenden Abrufs“ (so Rohdes Formulierung) für immer ins Totenreich verbannt. ${ }^{60}$

Diesem therapeutischen Konzept des Verhältnisses von Sprache und Vision ist Elektras feuerzüngige Rede genau entgegengesetzt. Sie setzt die Rede ein zur Beschwörung der Bilder. „Elektra mit ihrer Feuerzunge“, ein Satz aus Goethes Iphigenie, der nach Hofmannsthals Selbstaussage am Anfang seiner Auseinandersetzung mit dem Elektra-Stoff stand, ${ }^{61}$ ist im Stück immer wieder in Beziehung gesetzt zum züngelnden Fackelschein der aufzuckenden, durch die Beleuchtungsregie sinnlich erfahrbaren Schattenbilder. Elektra verweigert der unter ihren

55 Ebd., S. 66-68; Rohde: Psyche, Bd. 1, Kap. 1: ,Seelenglaube und Seelencult in den homerischen Gedichten', S. 1-67, hier S.19, Kap.5: ,Der Seelencult', S. 200-278. Die

Schilderung des Leichenbegängnisses des Patroklos aus dem 23. Gesang der Ilias findet sich bei Rohde S. 14-22. Vgl. Vogel: „Priesterin künstlicher Kulte“, S. 295-299.

56 Rohde: Psyche, Bd. 2, Kap. ,Der thrakische Dionysosdienst', S. 1-136, Zitate S. 11-13.

57 Ebd., Bd. 2, S.12.

58 Breuer u. Freud: Studien über Hysterie, S. 297 u. S. 50.

59 Ebd., S. 297.

60 Vgl. Rohde: Psyche, Bd.1, S. 9 f.

61 Hofmannsthal: „Elektra. Zeugnisse“. In: SW VII, S. 459. 
Visionen leidenden Mutter das „rechte Wort“, das sie zur Heilung vom Bann der Bilder verlangt. ${ }^{62}$ Auf die Aufforderung der Mutter: „Aber du hast Worte“63 , die sie zur magischen Herrin der Rede einsetzt: „[l]aß deine Zunge los“64, antwortet Elektra mit einer visionären Beschwörung des Opfertods der Mutter, ein Monolog, der nicht mit dem erlösenden Wort, sondern mit einem schweigenden „Gesicht“ endet:

[...] innen krampft es dich, / daß du von meinem schweigenden Gesicht / ein Wort ablesen willst, du rollst die Augen, / willst irgend etwas denken [...] /

[...] verendend willst du / dich auf ein Wort besinnen, irgend eines / noch von dir geben, nur ein Wort, anstatt / der blut'gen Träne, die dem Tier sogar / im Sterben nicht versagt ist: da steh' ich / vor dir, und nun liest du mit starrem Aug' / das ungeheure Wort, das mir in mein / Gesicht geschrieben ist: denn mein Gesicht / ist aus des Vaters und aus deinen Zügen / gemischt, und da hab' ich mit meinem stummen / Dastehn dein letztes Wort zunicht' gemacht, / [... $]^{65}$

Der Einzug des „letzten“ Wortes in der „gemischten“ Chimäre des Gesichts ist der eigentliche Gewaltakt, durch den Elektra die Worte $\mathrm{zu}$ Waffen verdinglicht. Elektras Rede spricht die Schreckbilder nicht therapeutisch ab, sondern beschwört sie im magischen Geisterbann herauf. Als solche geisterbeschwörende Schamanin, von den anderen als „Dämon“ bezeichnet, ${ }^{66}$ dem tierischen Bereich zugeordnet, verwahrlost im zu kurzen Gewand, tritt sie in der Eingangsvision des Opferfestes für den toten Vater auf. ${ }^{67}$ „Ich will dich sehn“, ruft sie das Eidolon des Vaters in dieser Prunkvision herbei, „[...] wie ein Schatten, dort / im Mauerwinkel

62 Vgl. Hofmannsthal: „Elektra“. In: SW VII, S. 85.

63 Ebd., S. 79.

64 Ebd., S. 78.

65 Ebd., S. 86.

66 Vgl. ebd., S. 64.

67 Zu Elektras Kleidung vgl. ebd., S. 100 u. Hofmannsthal: „Authentische Vorschriften für die Inscenierung“. In: ebd., S. 381: „Elektra trägt ein verächtliches elendes Gewand, das zu kurz für sie ist. Ihr Beine sind nackt, ebenso ihre Arme“. Das Tiermotiv wird bereits in der Eingangsszene in einer Häufung von Tierbildern eingeführt. Elektra „pfauchte [...] wie eine Katze“ (SW VII, S. 63), nennt die anderen „Schmeißfliegen“ (ebd., S. 63f.), sie „reckte ihre Fingeer / wie Krallen gegen uns und schrie: ,Ich füttre,'/schrie sie, ,mir einen Geier auf dem Leib. ““ (ebd., S. 64). Wie ein Hund liegt sie auf der Türschwelle oder gräbt in der Erde. Der Hund ist in Bachofens Mutterrecht, das Hofmannsthal in einer Ausgabe von 1897 wiederholt las, das chthonische Tier der Mutterwelt. (Bachofen, Johann Jakob: Das Mutterrecht. Eine Untersuchung über die Gynaikokratie der alten Welt nach ihrer religiösen und rechtlichen Natur. 2. Aufl. Basel 1897). Bei Rohde werden die Hunde als Begleiter der Hekate, der Herrin über die unsteten Seelen beschrieben. (Rohde: Psyche, Bd. 2, S. 83-86). 
zeig dich deinem Kind!“68 Der Fokus dieses Bilderbanns ist die Herstellung einer traumatischen Präsenz. In jeder ihrer Visionen wechselt das Tempus der autohypnotischen Rede plötzlich vom epischen Präteritum ins Präsens, das auch die antizipierte Zukunft der Erfüllung der Rache ins Hier und Jetzt einholt. Der Vater, der mit dem Kopf voran aus dem Zimmer getragen worden war, und also nach primitiver Vorstellung dieser falschen Stellung wegen mit den Füssen voran wieder zurückkommen wird, wie Rohde ausführt, kehrt in jeder dieser Visionen zurück. „So kommst du wieder, setzest Fuß vor Fuß / und stehst auf einmal da, [...]“. ${ }^{69}$ Die Zeitebenen fallen in Elektras Stunde, der Stunde einer langsam einsetzenden Dämmerung, wie die Regieanweisungen lauten, in eins. ${ }^{70}$ Dass Hofmannsthal das Stück nicht wie bei Sophokles mit dem Morgenlicht zum Tageslauf der Handlungstragödie anheben lässt, sondern es in das Zwielicht der Abenddämmerung Elektras „Stunde“ ${ }^{71}$ die Stunde der hypnoiden Delirien der Anna 0. - bannt, lässt die hysterische Fixierung der Hauptfigur auf eine klaustrophobische immerwährende Gegenwart auf die ganze Dramenstruktur übergreifen. Die Zeitfinalität der klassischen Tragödie ist durchkreuzt von einer anderen Zeiterfahrung, in der die Gleichzeitigkeit der Zeitebenen herrscht und die Elemente der dramatischen Welt nicht nach Ursache und Folge, sondern nach den analogischen Mechanismen einer halluzinatorischen Logik verknüpft sind. ${ }^{72}$

Unterstützt wird sie von der mit Max Reinhardt am kleinen Theater in Berlin umgesetzten Lichtregie, ${ }^{73} \mathrm{zu}$ der Hofmannsthal sowohl in den Regieanweisungen, als auch in den Authentischen Vorschriften zur Inscenierung sowie in dem Essay Die Bühne als Traumbild detaillierte Angaben machte. Hofmannsthal konzipierte die Bühne für sein Drama mit minimalistischen Mitteln, als eine Kombination aus klaustrophobisch verengtem Bühnenraum, der die Vorstellung eines engen Kerkers, einer „Nussschale“ aufrufen und darin das gefangene hypnoide Bewusstsein visualisieren soll, ${ }^{74}$ und dem „unerschöpflichen Spiel des Lichts“, das die My-

\footnotetext{
68 Hofmannsthal: „Elektra“. In: SW VII, S. 67.

69 Ebd.
}

70 Vgl. Hofmannsthal: „Authentische Vorschriften für die Inscenierung“. In: SW VII, S. 381.

71 Vgl. Hofmannsthal: „Elektra“. In: SW VII, S. 63.

72 So auch Vogel: „Priesterin künstlicher Kulte“, S. 287-306.

73 Vgl. Fiedler, Leonhard M.: „Die Überwindung des Naturalismus auf der Bühne: Das Theater Max Reinhardts“. In: Drama und Theater der Jahrhundertwende. Hg. v. Dieter Kafitz. Tübingen 1991, S. 69-85; ders. (Hg.): Der Sturm Elektra. Gertrud Eysoldt - Hugo von Hofmannsthal. Briefe. Wien 1996; Greiner, Bernhard: „,Damenopfer‘ für das Theater. Hofmannsthals und Reinhardts Begegnung in der Arbeit an Elektra“. In: Von Franzos zu Canetti. Jüdische Autoren aus Österreich. Neue Studien. Hg. v. H. Mark Gelber u. a. Tübingen 1996, S. $253-271$.

74 Vgl. Hofmannsthal: „Authentische Vorschriften für die Inscenierung“. In: SW VII, S. 379. 
thisierungen des halluzinierenden Blicks affirmiert. ${ }^{75}$ Das von zuckendem Fackellicht hervorgerufene Lichterspiel projiziert Schattenbilder an die Rückwand der Bühne, die als cineastischer screen fungiert. In den gerahmten Tür- und Fensteröffnungen dieser Welt erscheinen Bilder vor dem starren Auge Elektras, die im Dunklen steht und auf diese Wand starrt. Hier setzt Hofmannsthal die aus der Sinnesphysiologie entnommene Figur der Projektion um, die er in dem Text Die Bühne als Traumbild in der visionären Grundsituation der im Delirium zum Leben erwachten Bilder auf einer Kerkerwand geschildert hat. ${ }^{76}$ Daher folgt die Lichtregie den Projektionen Elektras und objektiviert sie zur cineastischen Projektion. Damit wird tendenziell die Unterscheidungsmöglichkeit zwischen der Realität der Bühnenhandlung und Elektras visionären Überblendungen unterminiert. Der Zuschauer wird gezwungen, den mythisierenden Dynamiken von Elektras Blick zu folgen. In der Eingangsvision sehen wir Elektra (so die Regieanweisung) „[...]allein mit den Flecken roten Lichtes, die aus den Zweigen des Feigenbaumes schräg über den Boden und auf die Mauern fallen, wie Blutflecke. "77 Niemand ist zugegen, der die animistische Deutung der Lichtflecken korrigieren könnte. Die Stunde der Abenddämmerung, mit der tief stehenden Sonne, ist die Stunde des Mythos. Ihr soll auch der Zuschauer erliegen, wie die Authentischen Vorschriften für die Inscenierung vorgeben:

Über das niedrige Dach des Hauses rechts wächst von draußen her ein riesiger schwerer gekrümmter Feigenbaum, dessen Stamm man nicht sieht, dessen Masse aber, unheimlich geformt im Abendlicht wie ein halbaufgerichtetes Thier, auf dem flachen Dach auflagert. ${ }^{78}$

Die Lichtmagie dient in Hofmannsthals Ästhetik der Verwischung der Grenzen von Identität und der Entdifferenzierung, welche die analogische Verwebung des Einzelnen in die „Welt der Bezüge“ leisten und in der Traumwelt der Bühne die Schranken zwischen den Geschöpfen als durchlässig erweisen soll. So entsteht ein Raum, in den das schöpferische Auge des Zuschauers im „unbestimmten, dunkelumhüllten Raum“79 im Zusammenspiel mit einer mythisierenden Phantasie Bedeutungen weben kann.

Eine solche Entdifferenzierung bestimmt auch die Individualität der drei weiblichen Hauptfiguren. Hofmannsthal nennt sie Ernst Hladny gegenüber

75 Hofmannsthal: „Die Bühne als Traumbild“. In: SW XXXIII, S. 41.

76 Ebd., S. 42.

77 Hofmannsthal: „Elektra“. In: SW VII, S. 66.

78 Hofmannsthal: „Authentische Vorschriften für die Inscenierung“. In: SW VII, S. 380.

79 Hofmannsthal: „Die Bühne als Traumbild“. In: SW XXXIII, S. 41. 
„Schattierungen eines intensiven und heimlichen Farbtones“. ${ }^{80}$ Die wechselseitigen Affinitäten, welche das Spiel des Lichts sichtbar macht als „antiindividuale [Kunst], eine Kunst des Flüssigmachens des Gestalteten“", ${ }^{81}$ affizieren diese drei Figuren wechselseitig. So sind alle drei Frauengestalten Somnambule, denen sich die Ebenen von Innen und Aussen vermischen. Der starre Blick Elektras scheint metonymisch von einer Frau zur nächsten weiter gewandert zu sein. Alle drei gehorchen derselben traumatischen Fixierung. Chrysothemis treibt es wie Elektra „immerfort im Hause herum“; wie Elektras hysterischer Körper sich im hysterischen Bogen stets in der Vertikale aufrichtet oder niederduckt, so bewegt sich auch Chrysothemis in der Vertikalen „treppauf, treppab“. ${ }^{82}$ Alle drei Frauengestalten sind zudem der Schwelle zugeordnet, die zwischen dem Innenraum und dem Aussenraum des Hauses und der zwischen „hier droben“, der Welt der Lebenden, und „da unten“, der Welt der Toten. ${ }^{83}$ Klytämnestras Bild erscheint im Tür- und Fensterrahmen, ${ }^{84}$ grell illuminiert, Elektras Ort ist die Türschwelle, ${ }^{85}$ von der sie sich erst in ihrem namenlosen Schlusstanz erhebt, und sowohl Chrysothemis als auch Elektra sind ,an den Boden [ge]schmiedet“86, wo Elektra hockt und dumpf nach unten ins chthonische Reich sprechend ,nach einer alten Leiche [scharrt]. “87 Alle drei Frauenfiguren haben zudem die Unterscheidung zwischen dem Reich der Lebenden und der Toten verloren. Während die Totenbilder sich gespenstisch verlebendigen, imaginieren die Frauen sich selbst als wesenlose SchattenbildSeelen. So nennt Elektra die Mägde Schmeissfliegen auf ihren Wunden, ${ }^{88}$ und zitiert damit aus Bachofens Mutterrecht eine Stelle, die sich Hofmannsthal in seinem Handexemplar angemerkt hatte. Die Schmeissfliege, so ist dort zu lesen, zeigt ,[i]n ihrer unabtreibbaren Schamlosigkeit und Lüsternheit nach Blut [...] den Menschen das unerbittliche Todesloos". ${ }^{89}$ Elektra nimmt sich also selbst als Leiche wahr, die sich als Schattenbild unter Schattenbildern bewegt. So auch Chrysothemis, die imaginiert, sie müsse die Seelenspeise eines ihr gewidmeten

80 Hofmannsthal: „Elektra. Zeugnisse“. In: SW VII, S. 459.

81 Vgl. Neumann, Carl: Rembrandt. 1. Bd. 2. vermehrte Aufl. Berlin, Stuttgart 1905, S. 529.

Auf derselben Seite befindet sich in Hofmannsthals Exemplar eine Anmerkung.

82 Hofmannsthal: „Elektra“. In: SW VII, S. 69.

83 Ebd., S. 98: „indes das Kind da unten in den Klüften des Grausens lungert [...]. Und ich hier droben allein!“

84 Ebd., S. 76.

85 Ebd., S. 65.

86 Ebd., S. 70.

87 Ebd., S. 64.

88 Ebd., S. $63 \mathrm{f}$.

89 Bachofen: Das Mutterrecht, S. $285 \mathrm{f}$. 
Totenopfers essen, ${ }^{90}$ und Klytämnestra, die sich ,lebendigen Leibes wie ein wüstes Gefild“ erfährt, aus der die „Nessel“ Elektra herauswächst. ${ }^{91}$ So wenig konturiert sind die Individualitäten der dramatis personae, dass sie chimärische Vermischungen eingehen. So in dem verwirrenden Tableau, von dem die Regieanweisung mitteilt:

[...] Elektra und Chrysothemis [liegen] aneinandergedrückt [...], wie ein Leib, den das Schluchzen der Chrysothemis schüttelt und über den sich das totenbleiche schweigende Gesicht der Elektra hebt. ${ }^{92}$

Das Drama endet mit Elektras schweigendem Tanz, der im kataleptischen Zusammenbruch kulminiert. Nicht die Erfüllung der in der Eingangsvision vorweggenommenen Verheißung bringt die Aufführung stummer Leiblichkeit im Schlusstableau, sondern Erstarrung und Tod..$^{93}$ Vollzogen wird damit die Einlösung einer das ganze Stück durchziehenden Erwartung, die Elektra ,in entsetzlicher Spannung“ gehalten hatte und alles zum „Merkzeichen“ dieses Schlusses funktionalisierte. ${ }^{94}$ Doch das auf Erwartung hin ausgerichtete infektiöse Bilderleben bricht am Schluss in sich zusammen. Das von katathymen Affektenergien gespeiste Bilderwesen führt daher auch nicht zur ersehnten Tat der Rache. „Ich habe ihm das Beil nicht geben können“, klagt Elektra am Schluss. ${ }^{95}$ Dass Elektra bis zum Schluss niemals die Schwelle von der Vision zur Tat überschreiten kann, lässt das als Racheszenario imaginierte Prunkopfer umkippen in ein Selbstopfer. Elektras flackernde Bilder gehorchen einer Logik der Selbstverzehrung, in ihrem letzten mänadischen Fackeltanz wird sie selbst zur Opferflamme. ${ }^{96}$ So spricht es Elektra Orest gegenüber aus:

90 Hofmannsthal: „Elektra“. In: SW VII, S. 72.

91 Ebd., S. 75.

92 Ebd., S. 89.

93 Vgl. Rutsch, Bettina: Leiblichkeit der Sprache. Sprachlichkeit des Leibes. Wort, Gebärde, Tanz bei Hugo von Hofmannsthal. Frankfurt a. M. 1998, S. 215-225.

94 Vgl. Hofmannsthal: „Elektra“. In: SW VII, S. 103 f. u. S. 106. Vgl. Schlötterer, Reinhold: „Elektras Tanz in der Tragödie Hugo von Hofmannsthals“. In: Hofmannsthal-Blätter 33 (1986), S. $47-58$.

95 Hofmannsthal: „Elektra“. In: SW VII, S. 106.

96 Zum Feuertanz der Elektra im Kontext der Tanzformen der Jahrhundertwende vgl. Brandstetter, Gabriele: Tanz-Lektüren. Körperbilder und Raumfiguren der Avantgarde. Frankfurt a. M. 1995, S. 275-278. 
[...] Haß ist nichts, er zehrt / und zehrt sich selber auf, und Liebe ist / noch weniger als Haß, sie greift nach allem / und kann nichts fassen, ihre Hände sind/ wie Flammen, die nichts fassen $[\ldots] .{ }^{97}$

Als solche sich selbst verzehrende Fackeln eines Opferbrandes imaginiert der fiktive Balzac, in dem Hofmannsthal den Inbegriff des Dichter-Visionärs mit all seinen Gefährdungen sah, am Schluss des kleinen poetologischen Gesprächs Ueber Charaktere im Roman und im Drama die ihrem Dämon ausgelieferten Dichter. ${ }^{98}$ Hofmannsthals primitivistische Poetik im Zeichen visionärer Präsenz thematisiert die ihr eigenen ästhetischen Risiken.

\section{Literaturverzeichnis}

Adorno, Theodor W.: „George und Hofmannsthal. Zum Briefwechsel: 1891-1906“. In: ders.: Prismen. Kulturkritik und Gesellschaft. Berlin, Frankfurt a. M. 1955, S. 232-282.

Bachofen, Johann Jakob: Das Mutterrecht. Eine Untersuchung über die Gynaikokratie der alten Welt nach ihrer religiösen und rechtlichen Natur. 2. Aufl. Basel 1897.

Bahr, Hermann: Dialog vom Tragischen. Berlin 1904.

Biese, Alfred: Die Philosophie des Metaphorischen. In Grundlinien dargestellt. Hamburg, Leipzig 1893.

Bohrer, Karl-Heinz: Das absolute Präsens. Die Semantik ästhetischer Zeit. Frankfurt a. M. 1994. Bohrer, Karl-Heinz: „Die Wiederholung des Mythos als Ästhetik des Schreckens. Hugo von Hofmannsthals Nachdichtung von Sophokles’ Elektra“. In: ders.: Das absolute Präsens. Die Semantik ästhetischer Zeit. Frankfurt a. M. 1994, S. 63-92.

Brandstetter, Gabriele: Tanz-Lektüren. Körperbilder und Raumfiguren der Avantgarde. Frankfurt a. M. 1995 , S. $275-278$.

Breuer, Josef u. Sigmund Freud: Studien über Hysterie (1895). Hg. v. Stavros Mentzos. Frankfurt a. M. 2000.

Fiedler, Leonhard M. (Hg.): Der Sturm Elektra. Gertrud Eysoldt - Hugo von Hofmannsthal. Briefe. Wien 1996.

Fiedler, Leonhard M.: „Die Überwindung des Naturalismus auf der Bühne: Das Theater Max Reinhardts“. In: Drama und Theater der Jahrhundertwende. Hg. v. Dieter Kafitz. Tübingen 1991, S. 69-85.

Frazer, James Georges: Der goldene Zweig. Das Geheimnis von Glauben und Sitten der Völker (engl. 1894). Aus dem Englischen von Helen von Bauer. Reinbek 1989.

Greiner, Bernhard: „,Damenopfer‘ für das Theater. Hofmannsthals und Reinhardts Begegnung in der Arbeit an Elektra“. In: Von Franzos zu Canetti. Jüdische Autoren aus Österreich. Neue Studien. Hg. v. Gelber, H. Mark u. a. Tübingen 1996, S. 253-271.

97 Hofmannsthal: „Elektra“. In: SW VII, S. 105.

98 Hofmannsthal: „Über Charaktere im Roman und im Drama. Ein imaginäres Gespräch“. In: SW XXXI, S. 27-39. 
Gumbrecht, Hans Ulrich: Diesseits der Hermeneutik. Die Produktion von Präsenz. Frankfurt a. M. 2004.

Helmholtz, Hermann: Handbuch der physiologischen Optik. Leipzig 1867.

Hofmannsthal, Hugo von: Gesammelte Werke in zehn Einzelbänden. Reden und Aufsätze I (1891-1913). Hg. v. Bernd Schoeller in Beratung mit Rudolf Hirsch. Frankfurt a. M. 1979 (Sigle RuA I).

Hofmannsthal, Hugo von: Gesammelte Werke in zehn Einzelbänden. Reden und Aufsätze III (1925-1929). Hg. v. Bernd Schoeller in Beratung mit Rudolf Hirsch. Frankfurt a. M. 1979 (Sigle RuA III).

Hofmannsthal, Hugo von: Sämtliche Werke. Kritische Ausgabe. Veranstaltet vom Freien Deutschen Hochstift. Hg. v. Rudolf Hirsch u. a. Bd. VII. Dramen 5. Hg. v. Klaus E. Bohnenkamp und Mathias Mayer. Frankfurt a. M. 1997 (Sigle SW VII).

Hofmannsthal, Hugo von: Sämtliche Werke. Kritische Ausgabe. Veranstaltet vom Freien Deutschen Hochstift. Hg. v. Rudolf Hirsch u.a. Bd. XXXI. Erfundene Gespräche und Briefe. Hg. v. Ellen Ritter. Frankfurt a. M. 1991 (Sigle SW XXXI).

Hofmannsthal, Hugo von: Sämtliche Werke. Kritische Ausgabe. Veranstaltet vom Freien Deutschen Hochstift. Hg. v. Rudolf Hirsch u. a. Bd. XXXIII. Reden und Aufsätze 2. Hg. v. Konrad Heumann und Konrad Ritter. Frankfurt a. M. 2011 (Sigle SW XXXIII).

Jäger, Ludwig: „Schauplätze der Evidenz: Evidenzverfahren und kulturelle Semantik. Eine Skizze“. In: Die Listen der Evidenz. Hg. v. Michael Cuntz u. a. Köln 2006, S. 37-52.

Krämer, Sybille: „Performanz - Aisthesis. Überlegungen zu einer aisthetischen Akzentuierung im Performanzkonzept“. In: Ereignis Denken. TheatRealität, Performanz, Ereignis. Hg. v. Arno Böhler u. Susanne Granzer. Wien 2009, S. 141-168.

Kretschmer, Ernst: Medizinische Psychologie. Ein Leitfaden für Studium und Praxis. Leipzig 1922.

Mersch, Dieter: Ereignis und Aura. Untersuchungen zu einer Ästhetik des Performativen. Frankfurt a. M. 2002.

Mersch, Dieter: Was sich zeigt. Materialität, Präsenz, Ereignis. München 2002.

Nancy, Jean Luc: The birth to presence. Stanford 1993.

Neumann, Carl: Rembrandt. 1. Bd. 2. vermehrte Aufl. Berlin, Stuttgart 1905.

Nietzsche, Friedrich: Sämtliche Werke. Kritische Studienausgabe in 15 Bänden. Hg. v. Giorgio Colli und Mazzino Montinari, Bd. 2. Menschliches, Allzumenschliches I und II. Neuausgabe. München 1999 (Sigle KSA 2).

Nietzsche, Friedrich: Sämtliche Werke. Kritische Studienausgabe in 15 Bänden. Hg. v. Giorgio Colli und Mazzino Montinari, Bd. 7. Nachgelassene Fragmente 1869-1874. Neuausgabe. München 1999.

Pater, Walter: The Renaissance. London, New York 1900.

Pfotenhauer, Helmut u. Sabine Schneider: Nicht völlig Wachen und nicht ganz ein Traum. Die Halbschlafbilder in der Literatur. Würzburg 2006.

Riedel, Wolfgang: „Arara ist Bororo oder die metaphorische Synthesis“. In: Anthropologie der Literatur. Poetogene Strukturen und ästhetisch-soziale Handlungsfelder. Hg. v. Rüdiger Zymner u. Manfred Engel. Paderborn 2004, S. 220-240.

Riedel, Wolfgang: „Archäologie des Geistes. Theorien des wilden Denkens um 1900“. In: Das schwierige neunzehnte Jahrhundert. Hg. v. Jürgen Barkhoff u. a. Tübingen 2000, S. 467-485.

Rohde, Erwin: Psyche. Seelencult und Unsterblichkeitsglaube der Griechen. 2 Bde. 3. Aufl. Tübingen, Leipzig 1903. 
Rutsch, Bettina: Leiblichkeit der Sprache. Sprachlichkeit des Leibes. Wort, Gebärde, Tanz bei Hugo von Hofmannsthal. Frankfurt a. M. 1998.

Schlötterer, Reinhold: „Elektras Tanz in der Tragödie Hugo von Hofmannsthals“. In: Hofmannsthal-Blätter 33 (1986), S. 47-58.

Schneider, Florian: „Augenangst? Die Psychoanalyse als ikonoklastische Poetologie“. In: Hofmannsthal-Jahrbuch 11 (2001), S. 197-240.

Schneider, Sabine: „Das Leuchten der Bilder in der Sprache. Hofmannsthals medienbewusste Poetik der Evidenz“. In: Hofmannsthal-Jahrbuch 11 (2003), S. 209-248.

Schneider, Sabine: „Die Welt der Bezüge. Hofmannsthal zur Autorität des Dichters in seiner Zeit“. In: Colloquium Helveticum 41 (2010), S. 203-221.

Schneider, Sabine: „Helldunkel - Elektras Schattenbilder oder die Grenzen der semiotischen Utopie“. In: dies.: Verheißung der Bilder. Das andere Medium in der Literatur um 1900. Tübingen 2006, S. $342-368$.

Schneider, Sabine: „Poetik der Illumination. Hugo von Hofmannsthals Bildreflexionen im Gespräch über Gedichte“. In: Zeitschrift für Kunstgeschichte 71 (2008), Heft 3, S. 389- 404.

Seel, Martin: Die Macht des Erscheinens. Texte zur Ästhetik. Frankfurt a. M. 2007.

Sommer, Manfred: Evidenz im Augenblick. Eine Phänomenologie der reinen Empfindung. Frankfurt a. M. 1996.

Treiber, Hubert: „Zur Logik des Traumes bei Nietzsche. Anmerkungen zu den Traum-Aphorismen aus Menschliches, Allzumenschliches“. In: Nietzsche-Studien 23 (1994), S. 1-41.

Tylor, Edward B.: Die Anfänge der Cultur. Untersuchungen über die Entwicklung der Mythologie, Philosophie, Religion, Kunst und Sitte. Unter Mitwirkung des Verfassers ins Deutsche übertragen v. J.W. Spengel u. Fr. Poske. 2 Bde. Leipzig 1873.

Tylor, Edward B.: Primitive Culture. Reasearch into the Development of Mythology, Philosophy, Religion, Art, and Custom. 2 Bde. London 1871.

Vischer, Friedrich Theodor: „Das Symbol“. In: ders.: Altes und Neues. Stuttgart 1889, S. $290-342$.

Vogel, Juliane: „Priesterin künstlicher Kulte. Ekstase und Lektüren in Hofmannsthals Elektra“. In: Tragödie - Idee und Transformation. Hg. v. Hellmut Falshar. Stuttgart u. a. 1997, S. 287-306.

Wellbery, David E.: „Die Opfer-Vorstellung als Quelle der Faszination. Anmerkungen zum Chandos-Brief und zur frühen Poetik Hofmannsthals“. In: Hugo von Hofmannsthal. Neue Wege der Forschung. Hg. v. Elsbeth Dangel-Pelloquin. Darmstadt 2007, S. 186-212.

Wiesing, Lambert: Artifizielle Präsenz. Studien zur Philosophie des Bildes. Frankfurt a. M. 2005. 